\title{
Reducing Versatile Bat Wing Conformations to a 1-DoF Machine
}

\author{
Jonathan Hoff ${ }^{1,2}$, Alireza Ramezani ${ }^{1}$, Soon-Jo Chung ${ }^{3}$, and Seth Hutchinson ${ }^{1,2}$ \\ 1 Coordinated Science Laboratory \\ 2 Department of Electrical and Computer Engineering \\ University of Illinois at Urbana-Champaign (UIUC) \\ Urbana, IL 61801, USA \\ ${ }^{3}$ Graduate Aerospace Laboratories (GAL) \\ California Institute of Technology \\ Pasadena, CA 91125, USA
}

\begin{abstract}
Recent works have shown success in mimicking the flapping flight of bats on the robotic platform Bat Bot (B2). This robot has only five actuators but retains the ability to flap and fold-unfold its wings in flight. However, this bat-like robot has been unable to perform folding-unfolding of its wings within the period of a wingbeat cycle, about $100 \mathrm{~ms}$. The DC motors operating the spindle mechanisms cannot attain this folding speed. Biological bats rely on this periodic folding of their wings during the upstroke of the wingbeat cycle. It reduces the moment of inertia of the wings and limits the negative lift generated during the upstroke. Thus, we consider it important to achieve wing folding during the upstroke. A mechanism was designed to couple the flapping cycle to the folding cycle of the robot. We then use biological data to further optimize the mechanism such that the kinematic synergies of the robot best match those of a biological bat. This ensures that folding is performed at the correct point in the wingbeat cycle.
\end{abstract}

Keywords: Aerial robotics, bats, biologically-inspired robots, kinematics

\section{Introduction}

Bats have a complex wing mechanism that has over 40 Degrees of Freedom (DoF) [16]. This structure allows them to actively fold and unfold their wings in the period of a wingbeat. It is thought that bats improve flight efficiency by folding their wings during the upstroke of the wingbeat cycle, which in turn reduces the moment of inertia of the wings [15]. Energy is thus saved by reducing the moment of inertia of the wings during the upstroke. Negative lift may be decreased as well. Bahlman et al. [1] studied the cost of flight by designing an articulated wing mechanism and testing both fixed wing positions and folding during the upstroke. Folding the wing did indeed lower the cost of flight: it used less power, and negative lift during the upstroke was reduced. While net thrust 
is reduced, negative lift is decreased (net lift is increased) and there is a decrease in power consumption because of the reduced inertial and aerodynamic costs.

Robots with flapping wings have shown success in this area of folding and unfolding the wings during flight. A one-way folding mechanism allowed for passive folding during the upstroke of a micro aerial vehicle (MAV) [9]. Similarly, Wissa et al. [19] reduced power consumption and improved lift of an ornithopter by inserting a compliant spine on the wings such that they were passively morphed during the upstroke. Another study designed wings that unfold in an outward sweeping motion from the centrifugal accelerations of wing flapping [18].

The folding of wings during the upstroke is an important aspect of bat flight. This capability was integrated into the design of the robotic Bat Bot (B2) in attempt to mimic the behavior of bat flight while maintaining a reduced complexity [11-14]. B2's morphing wings allow it to change its wing inertia, reduce wing area, and modulate the tension of the membrane.

In recent works, we presented an approach to match the kinematic synergies of a biological bat to those of B2 [7]. This entailed optimizing the geometric structure of the armwing as well as the actuator trajectories such that the synergies derived from principal component analysis (PCA) match those of the biological bat. Despite the drastic difference in DoFs, B2 was able to replicate the motion of flapping and folding-unfolding found in biological bats.

However, experimental testing showed that B2 could not match the rapid folding-unfolding of the armwing within the time interval of a wingbeat. The DC motors were incapable of driving the spindle mechanisms at the same frequency as the flapping motion. Thus, the wings could not be folded during the upstroke of the wingbeat period.

We address the hardware problem in this paper by coupling the flapping and folding motions of the wings with a four-bar crank-rocker mechanism. The new closed loop kinematic chain has one DoF operated by the main brushless DC motor (BLDC). This drives the flapping motion of the wings and now also operates the folding-unfolding motion of the wing. The methods in [7] are used to find the optimal dimensions of the designed mechanism and the actuator trajectory of the BLDC motor. The dimensions provide the best matching of the two most dominant principal components of B2 to those of a biological bat. We describe in detail the design process of the coupling mechanism in Sect. 2, optimization of the mechanism in Sect. 3, and the simulation results in Sect. 4. Concluding remarks are made in Sect. 5 .

\section{Mechanism design}

Systems of linkages are often designed to couple the motion of different mechanisms. This creates a synergy because there is coordinated movement. Synergies were first defined as cooperative activations of different muscle groups to simplify control for the central nervous system [2]. The human hand has over 20 DoFs [8], but many grasping tasks have DoFs that are coupled in movement. Research in grasping has found postural synergies in the human hand, i.e. move- 
ments in which joints are coupled in their behavior [17]. The two most dominant synergies have been implemented on a robotic hand using only two actuators and a system of pulleys [3]. A more recent study simplified the control of 19 DoFs with a single actuator using 'soft synergies' [4].

Studies in flapping flight have also attempted to reduce the number of actuators on aerial vehicles to reduce weight and add simplicity to the design. In a way, these are synergies, or couplings between the different movements. Coupling mechanisms have frequently been used for design of MAVs that mimic insect flight. A four-bar linkage was designed for an insect-like MAV to generate the figure eight pattern of the wing tip observed in insect flight using the planar figure eight pattern of the mechanism [20]. Conn et al. [5] designed a parallel crank-rocker mechanism with a single actuator that couples flapping and pitching. Pitching was adjusted by changing the phase of the parallel mechanism. The flight patterns of the wings of dragon flies were captured on a MAV using only one rotary actuator and a modified slider-crank mechanism such that wing rotations and flapping were coupled [6].

In a similar way, we considered the task of generating the folding-unfolding motion of the wing from the crank mechanism driving flapping. Currently, this motion of each wing is driven by a separate DC motor that moves a spindle mechanism. This in turn adjusts the position of B2's forelimb. The new mechanism should seamlessly integrate with the current crank design such that no modifications need to be made for the flapping configuration. In addition, the spindles and DC motors driving them should be replaced by sliders such that the folding can be driven by the crank mechanism. The mechanism should thus convert motion from the crank to linear motion of the new slider.

We used a four-bar linkage to convert the circular motion of the crank to motion of a rocker. The rocker arm in turn drives the slider (i.e. the spindle). This mechanical system has one DoF. The crank angle $q_{C}$ determines the complete motion of the flapping of $\mathrm{B} 2$ as well as the folding movement, replacing the two DC motors that drove the spindles. It should be noted that the DC motors can be introduced in cooperation with this coupling mechanism such that minor adjustments can be made in flight to the wing positions, though this will not be addressed in the paper.

The parameters defining the coupling mechanism are shown in Fig. 1. The shoulder joint $j_{0}$ is offset distances of $s_{y}$ and $s_{z}$ in the $-y$ and $z$ directions from the crank center $j_{3}$. The length of the crank radius is $r_{c}$. These are fixed parameters and will not be optimized. Link $l_{1}$ connects the end of the crank arm to the shoulder with ball-and-socket joints. Joint $j_{1}$ is a distance of $r_{s}$ from $j_{0}$.

The four-bar crank-rocker mechanism consists of the drive link $r_{c}$, the coupler link $l_{2}$, the rocker $l_{3}$, and the base dimension $\sqrt{w_{x}^{2}+w_{z}^{2}}$. Link $l_{2}$ couples the flapping motion produced by the crank arm to the rocker arm. Revolute joint $j_{4}$ is offset distances of $w_{x}$ and $w_{z}$ in the $-x$ and $-z$ directions from $j_{3}$.

The rocker arm is of length $l_{3}+l_{4}$. The end of the rocker $\left(j_{6}\right)$ is fixed to the slider at joint $j_{7}$ by link $l_{5}$. Ball-and-socket joints make up the two connections. Revolute joint $j_{8}$ is a distance $l_{6}$ from $j_{7}$ and is constrained along the slider in 


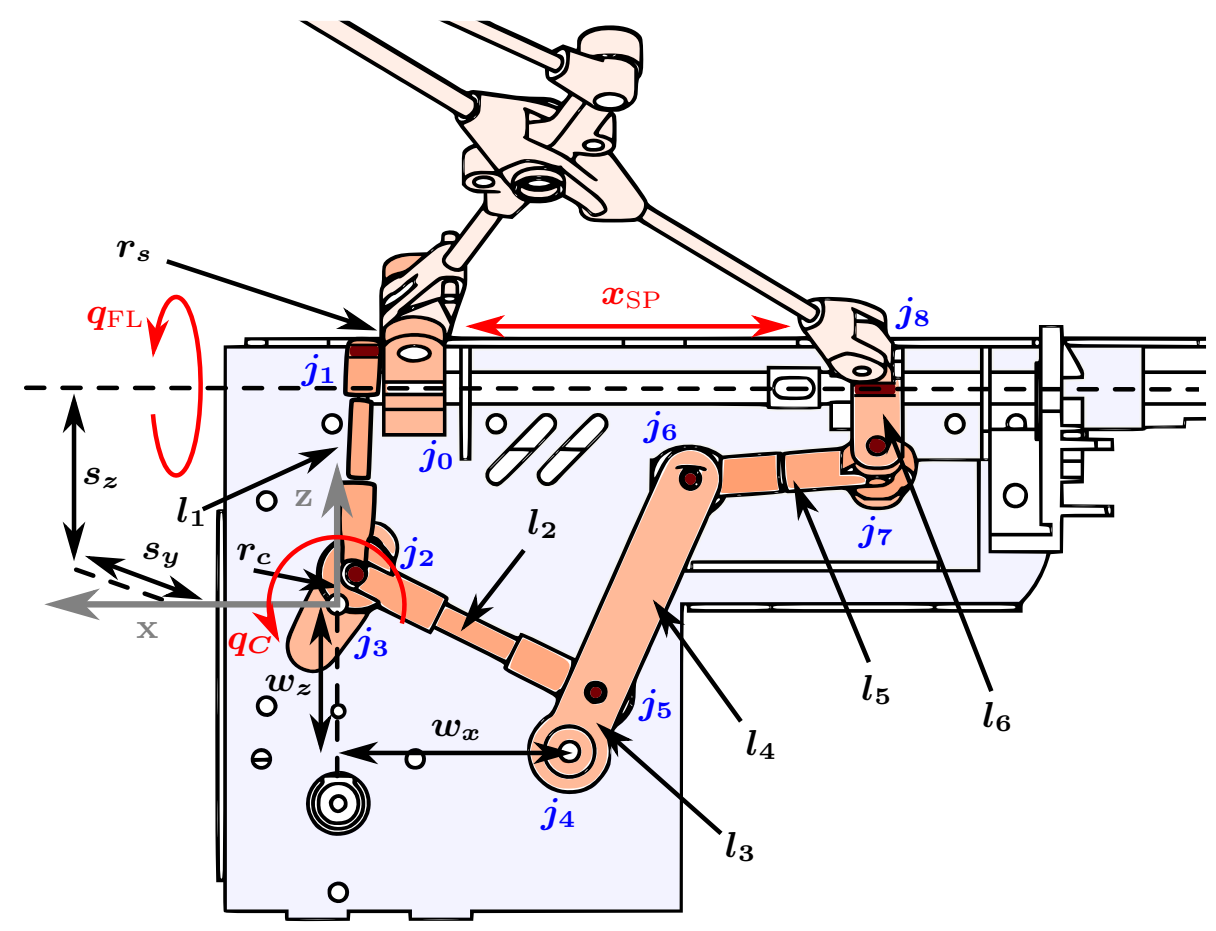

Fig. 1: Flapping and folding coupling mechanism. Red variables denote changing positions of angles and distance. Black variables give the parameters of the structure, and blue variables denote joint locations. Joints shaded brown experience both rotation and translation, and joints in white $\left(j_{0}, j_{3}\right.$, and $\left.j_{4}\right)$ rotate only. The coordinate system is colored gray, with the $\mathbf{y}$ axis pointing out of the page. This system has just one DoF: the crank angle $q_{C}$ is directly controlled by the BLDC motor, and this in turn characterizes the flapping angle $q_{\mathrm{FL}}$ and the spindle position $x_{\mathrm{SP}}$.

the $\pm x$ directions. Link $l_{6}$ can also rotate about the slider and is coupled to the flapping motion by the forelimb structure. All of the above listed lengths can be be combined to give the vector $\mathcal{C}$.

The mechanism has one DoF, and thus a set of kinematic constraints must be imposed to enforce this. The constraint equations for this mechanism can be solved analytically because it is a closed-loop kinematic chain and has one DoF. First, the constraint equations governing the linkage driving the flapping of the wing are solved to find the flapping angle $q_{\mathrm{FL}}$. This is the angle between the $\mathrm{xy}$ plane and the wing.

The link $l_{1}$ is projected onto the $\mathbf{x y}, \mathbf{x z}$, and $\mathbf{y z}$ planes, and the equations for these projections are combined as

$$
2 l_{1}^{2}=l_{1, x y}^{2}+l_{1, x z}^{2}+l_{1, y z}^{2}
$$


Algebraic manipulations produce the equation of form

$$
A \cos q_{\mathrm{FL}}+B \sin q_{\mathrm{FL}}+C \text {. }
$$

The terms $A, B$, and $C$ are functions of $q_{C}$ and $\mathcal{C}$. This is equivalent to Freudenstein's Equation, and thus $q_{\mathrm{FL}}$ can be solved for as a quadratic by making trigonometric substitutions for $\sin q_{\mathrm{FL}}$ and $\cos q_{\mathrm{FL}}$.

Next, the planar four-bar linkage shown in Fig. 1 can be solved. The drive link is $r_{c}$, and $q_{C}$ is the driving angle. The closed loop kinematics are written as

$$
\overrightarrow{j_{3} j_{2}}+\overrightarrow{j_{2} j_{5}}+\overrightarrow{j_{5} j_{4}}+\overrightarrow{j_{4} j_{3}}=0 .
$$

This means that the sum of the vectors around the linkage equals zero, i.e. the loop is constrained to be closed. This relation is also described by

$$
\operatorname{Rot}_{y}\left(\phi_{3}\right)\left[\begin{array}{c}
r_{c} \\
0 \\
0
\end{array}\right]+\operatorname{Rot}_{y}\left(\phi_{2}\right)\left[\begin{array}{c}
l_{2} \\
0 \\
0
\end{array}\right]+\operatorname{Rot}_{y}\left(\phi_{5}\right)\left[\begin{array}{c}
l_{3} \\
0 \\
0
\end{array}\right]+\operatorname{Rot}_{y}\left(\phi_{4}\right)\left[\begin{array}{c}
\|w\| \\
0 \\
0
\end{array}\right]=0,
$$

where $\|w\|=\sqrt{w_{x}^{2}+w_{z}^{2}}$ and $\phi_{2}, \phi_{3}, \phi_{4}$, and $\phi_{5}$ are the respective angular positions of joints $j_{2}, j_{3}, j_{4}$, and $j_{5}$. The angle $\phi_{4}$ is fixed at $\phi_{4}=2 \pi-\operatorname{atan} \frac{w_{z}}{w_{x}}$ and $\operatorname{Rot}_{y}$ is the rotation matrix about the $\mathbf{y}$ axis. Also, $\phi_{3}$ is equivalent to the crank angle $q_{C}$. These equations can be analytically solved by using algebraic manipulations to achieve the form of Eq. (2). The spindle position (or slider position) $x_{\mathrm{SP}}$ can then be determined from the resulting value of $\phi_{5}$. This is the distance between $j_{0}$ and $j_{8}$.

\subsection{Forelimb design [13]}

These equations give a mapping from $q_{C}$ and $\mathcal{C}$ to $q_{\mathrm{FL}}$ and $x_{\mathrm{SP}}$. From here, the forward kinematics in [7] give the positions of points on the forelimb of B2.

B2's forelimb is a three-link mechanism with a humeral link, a radial link, and a carpal plate. This mechanism is connected by revolute joints, and it is uniquely defined by three biologically meaningful angles: the shoulder retractionprotraction $q_{\mathrm{RP}}$, the elbow flexion-extension $q_{\mathrm{FE}}$, and the carpal plate abductionadduction $q_{\mathrm{AA}}$. This mechanism is constrained to one DoF by adding several extra links and fixing the radial link to the spindle.

The joint angles are directly controlled by the spindle position $x_{\mathrm{SP}}$, i.e. the distance between the shoulder joint and the radial link joint. Linear movements of the radial link joint along the spindle toward the shoulder result in forward rotation of the shoulder joint. This in turn forces the elbow to extend. The carpal plate is pushed away from the body in response to this extension. The digits of B2 are thin carbon fiber rods that are secured to the carpal plate, and these are pushed outward as a result of the movement of the carpal plate. These rods are flexible, and they introduce the passive DoFs of abduction-adduction and flexion-extension of the digits. The digits lack joints, and thus their motion is dependent on the movement of the carpal plate. The wing as a whole has one actuated DoF with several passive DoFs. This actuated DoF controls the three biologically meaningful angles. 


\section{Optimization [7]}

The dimensions of the coupling mechanism and forelimb as well as the trajectory of $q_{C}$ should be selected such that B2's kinematic motion best matches that of the biological bat. We use optimization to select the dimensions and the actuator trajectory. A brief summary of the methods are provided here. For a more thorough explanation, readers should see [7] as a reference. The optimization in this paper differs slightly from that presented in [7]: the structure of B2's forelimbs has been improved, marker selection was adjusted, the objective function was modified, and several extra constraints were added.

B2 was originally designed to mimic Rousettus aegyptiacus, though data for this bat was not available. In this study, we used data for Tadarida brasiliensis. Rousettus aegyptiacus is much larger than Tadarida brasiliensis, thus we linearly scaled the data for Tadarida brasiliensis such that its wingspan matches that of Rousettus aegyptiacus [10]. Additionally, the coordinate system is centered on the anterior sternum marker, the $\mathbf{x}$ axis passes through the two sternum markers, the $\mathbf{y}$ axis points to the left wing, orthogonal to gravity, and the $\mathbf{z}$ axis points up. This is the body-referenced coordinate system used by [16].

The shoulder marker and nine markers of the digits on the biological bat were selected for comparison. Complementary markers on B2 were chosen by projecting these biological bat markers onto the digits of B2 at each point in time over a wingbeat period to get the closest points on B2's digits to those on the biological bat's digits. These two sets of markers were then used in the optimization formulation.

The crank angle is parameterized based on the angular frequency $\omega$ and the phase $\phi$ as

$$
q_{C}\left(t_{i}\right)=\omega t_{i}+\phi
$$

These are combined into the vector $\mathcal{A}_{\mathrm{C}}=[\omega \phi]^{\top}$. Besides $l_{1}$, the crank assembly is not optimized. Crank radius $r_{c}$, shoulder radius $r_{s}$, and shoulder offsets $s_{y}$ and $s_{z}$ are left unchanged because the crank is already tuned to provide maximum torque for flapping while not overexerting the motor. Even small changes in lengths could lead to hardware issues. The rest of the parameters are optimized and are combined into the vector $\overline{\mathcal{C}}$.

The optimization routine for selected optimized variable $\mathcal{X}$ is formulated as a constrained nonlinear optimization problem to minimize the objective function

$$
\mathcal{J}(\mathcal{X})=\left\|\hat{\mathcal{M}}_{\mathrm{r}}(\mathcal{X})-\mathcal{M}_{\mathrm{r}}\right\|_{F}^{2} .
$$

Matrix $\mathcal{M}_{\mathrm{r}}$ is derived from the data matrix $\mathcal{M}$. The columns of $\mathcal{M}$ are the $x y z$ coordinates of each of the ten markers, and the rows are the time sample over a wingbeat cycle. We performed dimensionality reduction on $\mathcal{M}$ using PCA to use only the first two kinematic synergies (principal components) of the biological bat data, giving the matrix $\mathcal{M}_{\mathrm{r}}$. The matrix $\hat{\mathcal{M}}_{\mathrm{r}}$ is similarly derived for B2. The objective function implements a sum of squared differences between the points on B2 and the biological bat reconstructed from PCA using the Frobenius norm. 
The main optimization routine is separated into three subroutines in which the crank angle coefficients $\mathcal{A}_{\mathrm{C}}$, the mechanism parameters $\overline{\mathcal{C}}$, and the forelimb parameters $\overline{\mathcal{P}}$ are individually optimized. The vectors $\overline{\mathcal{C}}$ and $\overline{\mathcal{P}}$ contain only the parameters being optimized, whereas $\mathcal{C}$ and $\mathcal{P}$ contain all of the parameters describing the mechanism and the forelimb.

A set of inequality and equality constraints help shape the optimization problem. These constraints differ depending on the choice of $\mathcal{X}$. For $\mathcal{X}=\mathcal{A}_{\mathrm{C}}$, the equality constraint $q_{C}\left(t_{1}\right)-q_{C}\left(t_{n}\right) \in\{0, \pm 2 \pi, \pm 4 \pi, \cdots\}$ enforces periodicity of the motor cycle.

Several extra constraints are necessary for $\mathcal{X}=\overline{\mathcal{C}}$. First, the Grashof conditions for a four-bar mechanism are introduced. In order to drive the foldingunfolding motion, the crank must be able to spin freely, but the driving arm should be a rocker. Thus, the conditions for a crank-rocker mechanism were introduced as constraints to the optimization routine. These are given by the equations $-r_{4}-r_{2}+r_{1}+r_{3}<0,-r_{3}-r_{4}+r_{1}+r_{2}<0$, and $-r_{3}-r_{2}+r_{1}+r_{4}<0$, where $r_{1}=r_{c}, r_{2}=l_{2}, r_{3}=l_{3}$, and $r_{4}=\sqrt{w_{x}^{2}+w_{z}^{2}}$. In addition, the top of the rocker $\left(j_{6}\right)$ is restricted from passing above the shoulder line $\left(j_{0}-j_{8}\right)$, else it will interfere with the spindle mechanism and the membrane. The spindle position $x_{\mathrm{SP}}$ is also restricted to the range $x_{\min } \leq x_{\mathrm{SP}} \leq x_{\max }$, which is adjusted based on mechanical limitations. The mechanism parameters are restricted with upper and lower bounds as $l_{k} \leq \overline{\mathcal{C}}_{k} \leq u_{k}, k=1, \ldots, 8$.

Constraints are necessary for $\mathcal{X}=\overline{\mathcal{P}}$. The angles between the digits of B2 are forced not to overlap by the constraint $\gamma_{1} \leq \gamma_{2} \leq \gamma_{3}$. Additionally, the wing area of B2 is prevented from dropping below that of Rousettus aegyptiacus [10]. This is necessary such that the resulting structure of B2 can provide enough lift. The optimized variables also have upper and lower bounds as $l_{k} \leq \overline{\mathcal{P}}_{k} \leq u_{k}, k=$ $1, \ldots, 12$.

\section{Simulation Results}

We run the optimization routine separately for $q_{C}, \overline{\mathcal{C}}$, and $\overline{\mathcal{P}}$. The main optimization routine is iterated four times. The flapping angle trajectory $q_{\mathrm{FL}}$ can be compared to the biologically meaningful angles $q_{\mathrm{RP}}, q_{\mathrm{FE}}$, and $q_{\mathrm{AA}}$ describing the folding mechanism of the wing in order to observe the differences in phase and amplitude. Fig. 2 shows the evolution of these angles over a wingbeat period. The offset of each angle has been removed to provide a comparison of the phases and amplitudes of the trajectories. The angles $q_{\mathrm{RP}}$ and $q_{\mathrm{AA}}$ are in phase with each other as expected. They decrease when the wing is extending and increase when it is folding. The angle $q_{\mathrm{FE}}$ is $180^{\circ}$ out of phase, and thus moves opposite to these two angles. The three angles are out of phase with the flapping angle $q_{\mathrm{FL}}$. The wing initially extends at the very end of the upstroke to prepare for the downstroke, reaches maximum extension near the end of the downstroke, and begins to fold at the tail end of the downstroke and all through the upstroke.

The angles are also compared to those of the biological bat data in Fig. 3. First, the angles of the biological bat are centered about the origin by subtracting 


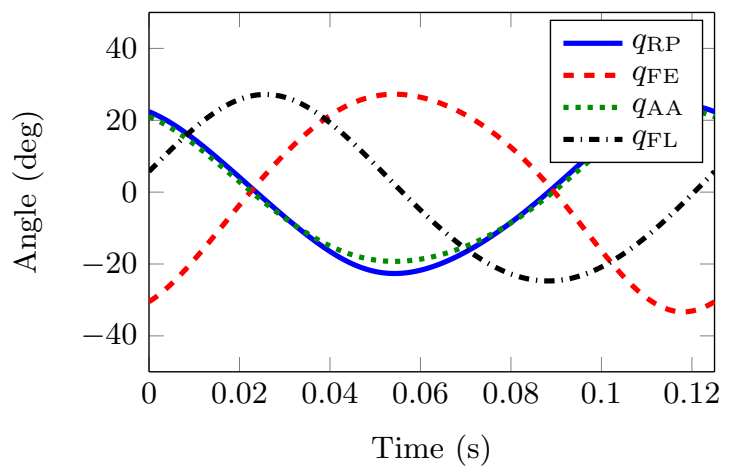

Fig. 2: Trajectories of the biologically meaningful angles $q_{\mathrm{RP}}, q_{\mathrm{FE}}, q_{\mathrm{AAA}}$, and $q_{\mathrm{FL}}$ over a wingbeat cycle. The trajectories have been centered about the origin to remove offsets in order to give a better comparison. These angles are all coupled by the motion of the crank angle $q_{C}$.

the mean from each. Second, for each angle, the trajectory is divided by its largest amplitude such that the trajectory remains between -1 and 1 . This is likewise done for B2's angles. The angles of B2 have much different offsets that those of the biological bat, and B2's angles have significantly larger amplitudes (except $q_{\mathrm{FL}}$, which has a lower amplitude). This is as expected because B2's forelimbs have a different topological structure than that of a biological bat due to the extra links added to constrain it. Thus, centering and normalizing the angles provides a way to characterize the changing behavior of the sets of angles on the same scale. The figure shows that the changing behavior of the flapping angle $q_{\mathrm{FL}}$ is almost identical to that of the biological bat, and the folding-unfolding motion is also comparable to this motion of the biological bat because of the matching of the angles $q_{\mathrm{RP}}$ and $q_{\mathrm{FE}}$. The wrist angles are less similar as there are high frequency oscillations of $q_{\mathrm{AA}}$ that are not present in B2. Though it cannot be seen in this figure, the flapping angle has a lower amplitude than the biological bat because the parameters that adjust the magnitude of the flapping angle are not optimized. As a result, the amplitude cannot increase to match the flapping amplitude of the biological bat. In practice, we have found that a larger amplitude results in hardware issues.

Fig. 4 gives the phase plots of the four angles for B2 and the biological bat. Angular velocities are generated from the position data by taking differences between adjacent angles and dividing by the sampling period. Similar to the above procedure, the angular positions and velocities are centered about the origin through mean subtraction and normalized between -1 and 1 . For the same reasons as above, this allows for better comparison of the behavior of position versus velocity of B2 and the biological bat even though it sacrifices magnitudes of these results. It can be seen from the phase plots that the states of B2 and the biological bat behave in a very similar manner, especially for the case of 


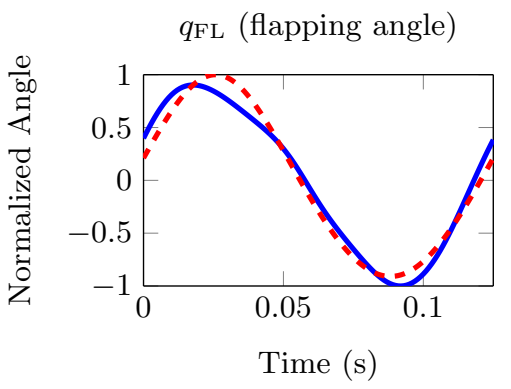

$q_{\mathrm{FE}}($ elbow angle)

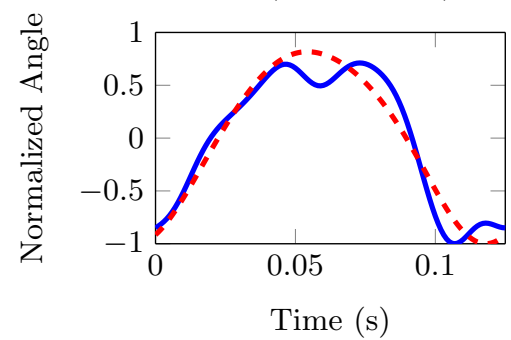

$q_{\mathrm{RP}}$ (shoulder angle)

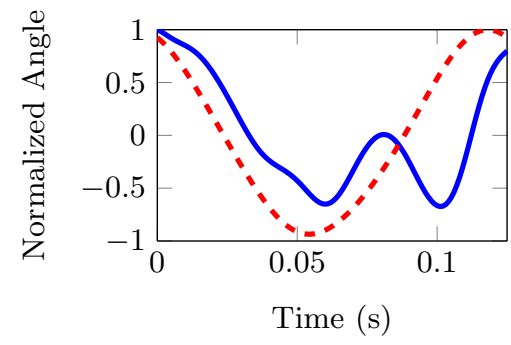

$q_{\mathrm{AA}}$ (wrist angle)

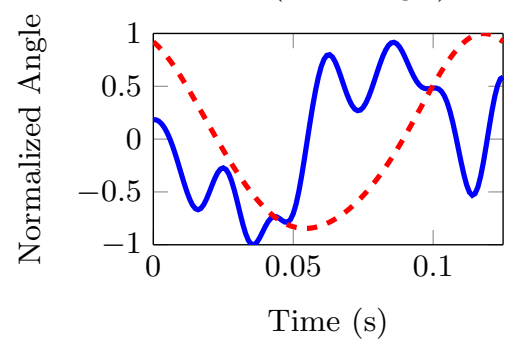

Fig. 3: Normalized biologically meaningful angles of B2 are compared to those of the biological bat over one wingbeat cycle. These angles are centered about the origin, and then normalized between -1 and 1 , allowing for better comparison between the biological bat's angles (blue lines) and B2's angles (dotted red lines). The amplitudes of B2's angles (except $q_{\mathrm{FL}}$ ) are significantly larger than the biological bat's when not normalized. All of these angles are coupled in B2 and move in response to the crank position $q_{C}$.

$q_{\mathrm{FL}}$. The wrist angle $q_{\mathrm{AA}}$ of the biological bat has several oscillations within the wingbeat cycle. This is also present in $q_{\mathrm{RP}}$ and $q_{\mathrm{FE}}$ but less pronounced.

Information can also be gained by analyzing the results from PCA. The principal components themselves give the directions of motion of each point on the wing. When considering only one component, the point is constrained to movement on a line. When considering two components, the point resides in a space spanned by the two components. Thus the point can move in two dimensions by taking linear combinations of the principal components. Different directions of motion are determined by taking different weights of the two components and adding them together. These weights are equivalent to the projection of the original data onto the principal components.

This is realized in Fig. 5. The data markers from the biological bat are projected onto the principal components to give the temporal weights over time, i.e. how the magnitudes of the principal components vary over the course of a wingbeat cycle. The plot shows the evolution of the weights of the two most dominant principal component over a wingbeat. We can further understand the relation between flapping and folding here because the first component is equivalent to the 

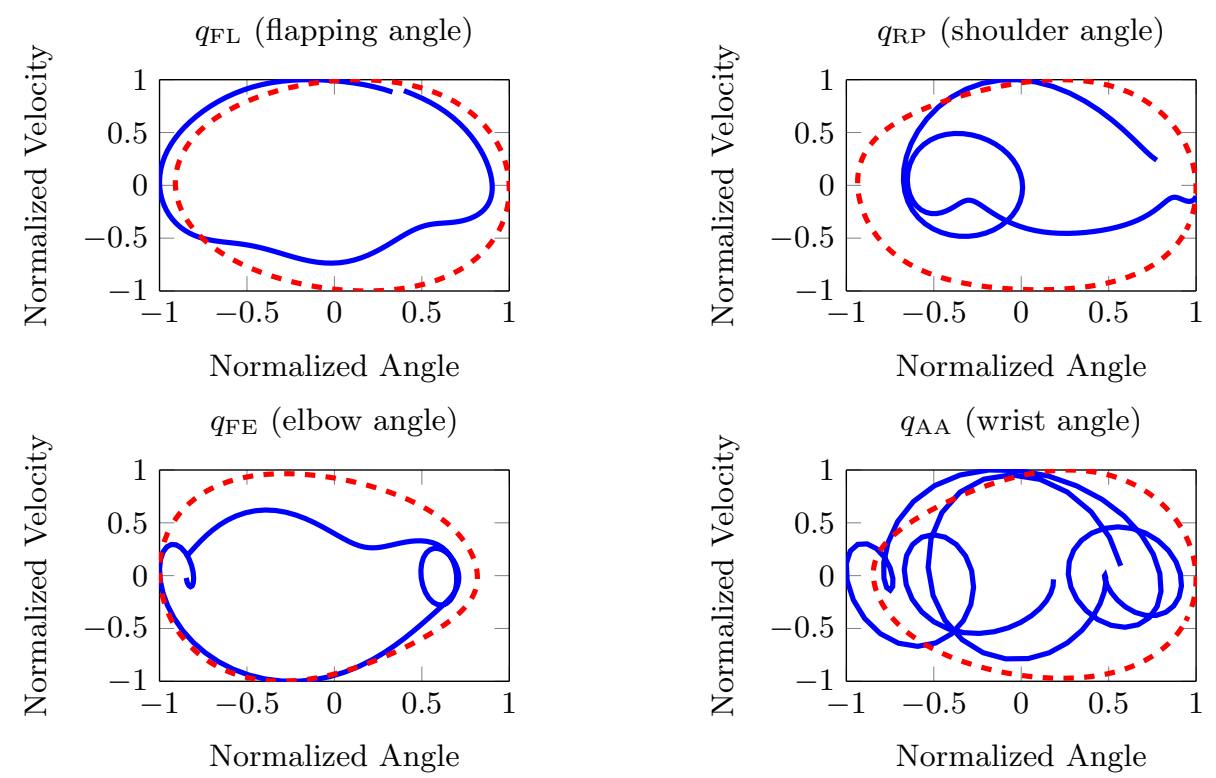

Fig. 4: Normalized phase plots of angular velocities versus angular positions of the four biologically meaningful angles in B2 and the biological bat. These angular positions and velocities are centered about the origin, and then normalized between -1 and 1, allowing for better comparison between the phase plots of the biological bat (blue lines) and B2 (dotted red lines). The amplitudes of B2's angles (except $q_{\mathrm{FL}}$ ) are significantly larger than the biological bat's when not normalized.

direction of flapping, and the second gives the direction of the folding-unfolding of the wing. Studying these weights of these two directions shows how far the wing folds at some certain point in the flapping cycle. Even though flapping and folding are coupled, the resulting motion is still quite similar to the behavior of the biological bat.

\section{Conclusion}

Coupling the flapping and folding-unfolding motions of a robotic bat with a mechanism can yield behavior that is consistent with that found in biological bats. The dimensions of this mechanism have been selected via optimization such that the basic kinematic synergies of wing flapping and folding-unfolding of the robot closely match those of a biological bat. The folding-unfolding of the wing can be parameterized based on the flapping angle because the two motions are coupled, and thus the flapping angle alone can give the position of the wing.

We have reduced the DoFs of the forelimbs of B2 from three to one by introducing this coupling mechanism. This further simplifies the design of the 


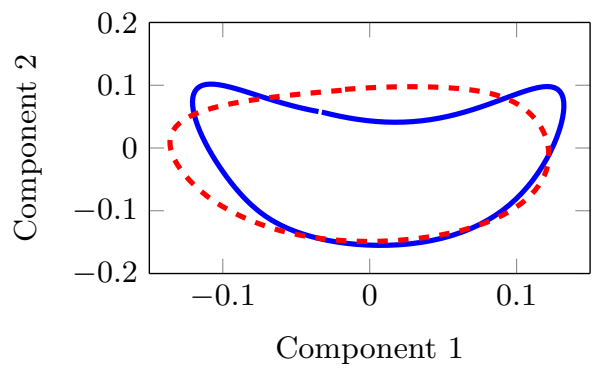

Fig. 5: Marker position data for both the biological bat and B2 are projected onto the two most dominant principal components. Component 1, the flapping direction, is plotted against component 2, the folding-unfolding direction. B2 is shown in red, and the biological bat is blue.

robot, but it removes control inputs that are helpful in stabilizing B2 during flight and are necessary for banking maneuvers. The controller makes adjustments to the spindle positions during flight to reduce or increase the surface area of the wings in order to improve roll stability. The hindlimbs can also stabilize for roll, but they have more significant effects on the pitch angle of B2.

However, future modifications could allow for the mechanism to couple flapping and folding as well as for the DC motors to make spindle adjustments during flight. This would ensure folding-unfolding within a wingbeat as well as independent control of each spindle to adjust the wings.

Acknowledgments. We would like to thank the team of graduate and undergraduate students from aerospace, electrical, computer, and mechanical engineering departments at the University of Illinois at Urbana-Champaign for their contribution to construct the initial prototype of B2.

The biological motion capture data set was provided by Dr. Kenneth Breuer and Dr. Sharon Swartz from Brown University. We would like to thank them in their assistance with this, as well as José Iriarte-Díaz for compiling the data.

This work was supported by NSF Grant 1427111.

\section{References}

1. J. W. Bahlman, S. M. Swartz, and K. S. Breuer. Design and characterization of a multi-articulated robotic bat wing. Bioinspiration \& Biomimetics, 8(1):016009, 2013.

2. N. A. Bernstein. The coordination and regulation of movements. Pergamon Press, Oxford, 1967.

3. C. Y. Brown and H. H. Asada. Inter-finger coordination and postural synergies in robot hands via mechanical implementation of principal components analysis. IEEE/RSJ International Conference on Intelligent Robots and Systems (IROS), pages $2877-2882,2007$. 
4. M. G. Catalano, G. Grioli, E. Farnioli, A. Serio, C. Piazza, and A. Bicchi. Adaptive synergies for the design and control of the Pisa/IIT SoftHand. The International Journal of Robotics Research, 33(5):768-782, 2014.

5. A. Conn, S. Burgess, and C. Ling. Design of a parallel crank-rocker flapping mechanism for insect-inspired micro air vehicles. Proceedings of the Institution of Mechanical Engineers, Part C: Journal of Mechanical Engineering Science, 221(10):1211-1222, 2007.

6. M. A. Fenelon and T. Furukawa. Design of an active flapping wing mechanism and a micro aerial vehicle using a rotary actuator. Mechanism and Machine Theory, 45(2):137-146, 2010.

7. J. Hoff, A. Ramezani, S.-J. Chung, and S. Hutchinson. Synergistic design of a bioinspired micro aerial vehicle with articulated wings. Robotics: Science and Systems (RSS), 2016.

8. J. Lin, Y. Wu, and T. S. Huang. Modeling the constraints of human hand motion. IEEE Workshop on Human Motion, pages 121-126, 2000.

9. D. Mueller, J. W. Gerdes, and S. K. Gupta. Incorporation of passive wing folding in flapping wing miniature air vehicles. ASME Mechanism and Robotics Conference, pages 797-805, 2009.

10. U. M. Norberg and J. M. Rayner. Ecological morphology and flight in bats (Mammalia; Chiroptera): wing adaptations, flight performance, foraging strategy and echolocation. Philosophical Transactions of the Royal Society B: Biological Sciences, 316(1179):335-427, 1987.

11. A. Ramezani, S.-J. Chung, and S. Hutchinson. A biomimetic robotic platform to study flight specializations of bats. Science Robotics, 2(3), 2017.

12. A. Ramezani, X. Shi, S.-J. Chung, and S. Hutchinson. Lagrangian modeling and flight control of articulated-winged bat robot. IEEE/RSJ International Conference on Intelligent Robots and Systems (IROS), pages 2867-2874, 2015.

13. A. Ramezani, X. Shi, S.-J. Chung, and S. Hutchinson. Bat Bot (B2), a biologically inspired flying machine. IEEE International Conference on Robotics and Automation (ICRA), pages 3219-3226, 2016.

14. A. Ramezani, X. Shi, S.-J. Chung, and S. Hutchinson. Modeling and nonlinear flight controller synthesis of a bat-inspired micro aerial vehicle. AIAA Guidance, Navigation, and Control Conference, page 1376, 2016.

15. D. K. Riskin, A. Bergou, K. S. Breuer, and S. M. Swartz. Upstroke wing flexion and the inertial cost of bat flight. Proceedings of the Royal Society of London B: Biological Sciences, 279(1740):2945-2950, 2012.

16. D. K. Riskin, D. J. Willis, J. Iriarte-Díaz, T. L. Hedrick, M. Kostandov, J. Chen, D. H. Laidlaw, K. S. Breuer, and S. M. Swartz. Quantifying the complexity of bat wing kinematics. Journal of Theoretical Biology, 254(3):604-615, 2008.

17. M. Santello, M. Flanders, and J. F. Soechting. Postural hand synergies for tool use. The Journal of Neuroscience, 18(23):10105-10115, 1998.

18. A. K. Stowers and D. Lentink. Folding in and out: passive morphing in flapping wings. Bioinspiration \& Biomimetics, 10(2):025001, 2015.

19. A. Wissa, Y. Tummala, J. Hubbard Jr, and M. Frecker. Passively morphing ornithopter wings constructed using a novel compliant spine: design and testing. Smart Materials and Structures, 21(9):094028, 2012.

20. R. Zbikowski, C. Galinski, and C. B. Pedersen. Four-bar linkage mechanism for insectlike flapping wings in hover: Concept and an outline of its realization. Transactions of the ASME: Journal of Mechanical Design, 127(4):817-824, 2005. 